\title{
Symmetric hyperbolic systems for Bianchi equations
}

\author{
Miguel Á.G. Bonilla* \\ Departament de Física Fonamental, Universitat de Barcelona, \\ Av. Diagonal 647, E-08028 Barcelona, Spain \\ E-mail: mangel@ffn.ub.es
}

August 7, 2021

\begin{abstract}
We obtain a family of first-order symmetric hyperbolic systems for the Bianchi equations. They have only physical characteristics: the light cone and timelike hypersurfaces. In the proof of the hyperbolicity, new positivity properties of the Bel tensor are used.
\end{abstract}

PACS number: 04.20.Ex

\section{Introduction}

First-order symmetric hyperbolic (FOSH) systems have recently been the subject of intensive research, not only for their theoretical interest in the Cauchy problem, but also for their applications to numerical relativity [1]. Such systems constitute a powerful procedure that can be used to establish a well-posed initial-value formulation for physical systems, since most of them can be cast into this FOSH form [2] and, within this framework, theorems of existence and uniqueness of solutions are applicable [3] (see also (田). More particularly, the Bianchi identities (investigated for a long time mainly for its usefulness in the study of the gravitational field propagation) are the basis from where some FOSH systems have been extracted (see [5] and references therein). Many of these results have been obtained by making use of the vacuum Bianchi identities, however the non-vacuum ones can also be applied to the construction of FOSH systems for the Einstein field equations [6].

In this paper a family of FOSH systems for the Bianchi equations is constructed. They may be useful in deriving new hyperbolic systems for Einstein's field equations, analogously to what is done in [6], where Bianchi identities are linked to equations for the spatial metric and connection. We begin in section 2 by considering the Bianchi

${ }^{*}$ Also at Laboratori de Física Matemàtica, Societat Catalana de Física, IEC, Barcelona 
equations applied to a double 2-form which does not satisfy a priori the additional Riemann symmetries. From these equations we extract a FOSH system of evolution equations having no unphysical characteristics. Actually, their only characteristics are the null cone and timelike hypersurfaces. In section 3 we briefly investigate the set of constraints that completes the hyperbolic system found in section 2. Both together, the evolution equations and the constraints, are equivalent to the original Bianchi equations. Finally, in section 4 we show that the full Riemann symmetries (together with the Einstein field equations) are conserved under the evolution. In the appendix we prove new positivity properties of the Bel tensor that are needed for the proof of the hyperbolicity.

\section{Hyperbolizations of Bianchi equations}

We recall that the Bianchi identities for the Riemann tensor read:

$$
\nabla_{[\nu} R_{\alpha \beta] \lambda \mu}=0
$$

from where it follows (by contracting, using the Riemann symmetries and the Einstein field equations with $8 \pi G=c=1)$ :

$$
\nabla^{\beta} R_{\alpha \beta \lambda \mu}=2 \nabla_{[\mu}\left(T_{\lambda] \alpha}-\frac{1}{2} T g_{\lambda] \alpha}\right) \equiv J_{\alpha \lambda \mu}
$$

$T_{\alpha \beta}$ being the energy-momentum tensor. For the system (1, 2), called the superior order field equations by Lichnerowicz, Einstein's field equations can be considered as initial data |7]|: if they hold at the initial spatial hypersurface $S$, they also hold in a neighborhood of $S$. In the last section we will see that the symmetries can also be taken as initial data.

Consider now the set of equations (1, 2), which will be called Bianchi equations in what follows, applied to a double 2-form $K_{\alpha \beta \lambda \mu}=K_{[\alpha \beta][\lambda \mu]}$. We do not suppose it to

have the additional Riemann symmetries $K_{[\alpha \beta \lambda] \mu}=0$ at the present moment. The Bianchi equations for $K_{\alpha \beta \lambda \mu}$ are then:

$$
\begin{gathered}
\nabla^{\beta} K_{\alpha \beta \lambda \mu}=J_{\alpha \lambda \mu}, \\
\nabla_{[\nu} K_{\alpha \beta] \lambda \mu}=0 .
\end{gathered}
$$

This is an overdetermined linear first-order system of 48 equations for 36 unknowns which is not manifestly symmetric hyperbolic. To handle more easily equations (3,4), it is better to write them in the following compact form (in a similar way to what is done in [2] for other systems):

$$
A_{I}^{\epsilon \gamma \delta \sigma \rho} \nabla_{\epsilon} K_{\gamma \delta \sigma \rho}=j_{I}
$$

where the index $I$ lives in the space of equations, $I=(\alpha[\lambda \mu],[\nu \alpha \beta][\lambda \mu])$, and

$$
A_{I}^{\epsilon \gamma \delta \sigma \rho}=\left(\delta^{\epsilon[\delta} \delta_{\alpha}^{\gamma]} \delta_{[\lambda}^{[\sigma} \delta_{\mu]}^{\rho]}, \delta_{[\nu}^{\epsilon} \delta_{\alpha}^{[\gamma} \delta_{\beta]}^{\delta]} \delta_{[\lambda}^{[\sigma} \delta_{\mu]}^{\rho]}\right), \quad j_{I}=\left(J_{\alpha \lambda \mu}, 0\right) .
$$


A tensor $H^{I} \gamma^{\prime} \delta^{\prime} \sigma^{\prime} \rho^{\prime}$ is said to be a hyperbolization of (5) if the matrix $Q^{\epsilon}\left\{\gamma^{\prime} \delta^{\prime} \sigma^{\prime} \rho^{\prime}\right\}\{\gamma \delta \sigma \rho\} \equiv$ $H^{I} \gamma^{\prime} \delta^{\prime} \sigma^{\prime} \rho^{\prime} A_{I}^{\epsilon} \gamma \delta \sigma \rho$ acting over the space of double 2 -forms satisfies the following properties: $i)$ it is symmetric, and $i i)$ there exists a 1 -form $n_{\epsilon}$ such that $n_{\epsilon} \mathbf{Q}^{\epsilon}$ is positivedefinite. By means of such a hyperbolization, we can transform (5) into:

$$
H^{I \gamma \delta \sigma \rho} A_{I}^{\epsilon \gamma^{\prime} \delta^{\prime} \sigma^{\prime} \rho^{\prime}} \nabla_{\epsilon} K_{\gamma^{\prime} \delta^{\prime} \sigma^{\prime} \rho^{\prime}}=H^{I \gamma \delta \sigma \rho} j_{I}
$$

which is now a FOSH system and, therefore, it admits the theorems of existence and uniqueness of solutions.

The set of 1-forms $n_{\epsilon}$ satisfying property ii) has a clear physical meaning (see [2] for instance) related to the propagation of the physical fields involved and to the causal character of the system. In fact, a FOSH system is said to be causal if every $n_{\epsilon}$ satisfying ii) is a future-directed 1-form.

For the Bianchi equations (5) the following family of tensors are hyperbolizations:

$$
\begin{array}{r}
H^{I \gamma^{\prime} \delta^{\prime} \sigma^{\prime} \rho^{\prime}}=\left(\delta^{\alpha\left[\gamma^{\prime}\right.} t^{\left.\delta^{\prime}\right]} h^{[\lambda \mu]\left[\sigma^{\prime} \rho^{\prime}\right]},-\frac{3}{2} t^{[\nu} \delta^{\alpha \mid\left[\gamma^{\prime}\right.} \delta^{\left.\left.\delta^{\prime}\right] \mid \beta\right]} h^{[\lambda \mu]\left[\sigma^{\prime} \rho^{\prime}\right]}\right) \\
h^{\lambda \mu \sigma^{\prime} \rho^{\prime}}=g^{\lambda \sigma^{\prime}}\left[u^{(\mu} v^{\left.\rho^{\prime}\right)}-\frac{1}{4}\left(u_{\nu} v^{\nu}\right) g^{\mu \rho^{\prime}}\right]
\end{array}
$$

where $\vec{t}, \vec{u}$ and $\vec{v}$ are arbitrary timelike future-directed vectors. To check property $i)$ we compute, for any two double 2 -forms $S_{\gamma \delta \sigma \rho}$ and $T_{\gamma \delta \sigma \rho}$, the quantity

$$
\begin{array}{r}
Q^{\epsilon} \gamma_{\left.\gamma^{\prime} \delta^{\prime} \sigma^{\prime} \rho^{\prime}\right\}\{\gamma \delta \sigma \rho\}} S_{\gamma^{\prime} \delta^{\prime} \sigma^{\prime} \rho^{\prime}} T_{\gamma \delta \sigma \rho}= \\
=\left[\frac{1}{2}\left(S_{\nu}^{\alpha \beta}{ }_{\sigma} T_{\alpha \mu \beta \rho}+T_{\nu}^{\alpha \beta}{ }_{\sigma} S_{\alpha \mu \beta \rho}+S_{\nu}^{\alpha \beta}{ }_{\rho} T_{\alpha \mu \beta \sigma}+T_{\nu}^{\alpha \beta}{ }_{\rho} S_{\alpha \mu \beta \sigma}\right)-\right. \\
-\frac{1}{4} g_{\nu \mu}\left(S_{\sigma}^{\alpha \beta \gamma} T_{\alpha \beta \gamma \rho}+T_{\sigma}^{\alpha \beta \gamma} S_{\alpha \beta \gamma \rho}\right)-\frac{1}{4} g_{\sigma \rho}\left(S_{\nu}{ }^{\alpha \beta \gamma} T_{\mu \alpha \beta \gamma}+T_{\nu}{ }^{\alpha \beta \gamma} S_{\mu \alpha \beta \gamma}\right)+ \\
\left.+\frac{1}{8} g_{\nu \mu} g_{\sigma \rho} S_{\alpha \beta \gamma \delta} T^{\alpha \beta \gamma \delta}\right] g^{\nu \epsilon} t^{\mu} u^{\sigma} v^{\rho}=Q^{\epsilon\left\{\gamma^{\prime} \delta^{\prime} \sigma^{\prime} \rho^{\prime}\right\}\{\gamma \delta \sigma \rho\}} T_{\gamma^{\prime} \delta^{\prime} \sigma^{\prime} \rho^{\prime}} S_{\gamma \delta \sigma \rho},
\end{array}
$$

from where the symmetry is clear. Property ii) is demonstrated in the appendix by proving that $n_{\epsilon} \mathrm{Q}^{\epsilon}$ is a positive-definite quadratic form for any $n_{\epsilon}$ timelike futuredirected 1-form. This also shows that the system is causal.

Let us now study the characteristics of the hyperbolized Bianchi equations found above, which finally read:

$$
h_{\alpha \beta}^{[\sigma \rho]}\left(t^{[\delta} \nabla_{\nu} K^{\gamma] \nu \alpha \beta}-\frac{3}{2} t_{\epsilon} \nabla^{[\epsilon} K^{\gamma \delta] \alpha \beta}\right)=h_{\alpha \beta}^{[\sigma \rho]}\left(t^{[\delta} J^{\gamma] \alpha \beta}\right) .
$$

Recall that a 1 -form $\zeta_{\alpha}$ is, by definition, characteristic for the system (6) if $\operatorname{det}\left(\zeta_{\epsilon} \mathbf{Q}^{\epsilon}\right)=$ 0 . To study the kernel of the characteristic matrix, first notice that $h_{[\alpha \beta][\lambda \mu]}$ (considered as a matrix acting over 2 -forms) has non-zero determinant, that is:

$$
h_{[\alpha \beta][\lambda \mu]} A^{\lambda \mu}=0 \Longrightarrow A^{[\lambda \mu]}=0 .
$$


This can be easily proven by contracting (7) successively with $\vec{u}$ and $\vec{v}$. Therefore,

$$
Q^{\epsilon\left\{\gamma^{\prime} \delta^{\prime} \sigma^{\prime} \rho^{\prime}\right\}\{\gamma \delta \sigma \rho\}} \zeta_{\epsilon} K_{\gamma \delta \sigma \rho}=0 \Longleftrightarrow t^{[\delta} \zeta_{\nu} K^{\gamma] \nu \alpha \beta}-\frac{3}{2} t_{\epsilon} \zeta^{[\epsilon} K^{\gamma \delta] \alpha \beta}=0 .
$$

The non-trivial solutions of this system are found to be of two types: i) $\zeta_{\alpha} \zeta^{\alpha}=0$ and ii) $\zeta_{\alpha} t^{\alpha}=0$. In the first case, the solutions are the double 2-forms $K_{\alpha \beta \lambda \mu}$ satisfying $\zeta^{\alpha} K_{\alpha \beta \lambda \mu}=0$ and $\zeta_{[\nu} K_{\alpha \beta] \lambda \mu}=0$. In the second one the solutions are those $K_{\alpha \beta \lambda \mu}$ having in its two first indices the structure $A(t \wedge \zeta)+B *(t \wedge \zeta)$ ( "** is the usual dual operator). Therefore, as remarked above, (6) has only physical characteristics: $i$ ) the light cone and ii) timelike hypersurfaces.

\section{Equivalence with the original system}

From the Bianchi equations we have constructed the 36 propagation equations (6) (in the sense that they contain derivatives in any timelike direction $n_{\alpha}$ ). In this section we will show that these equations, when completed with the constraints, are equivalent to the original system (3, (4). Taking $n_{\nu}$ as the normal to an initial-value spatial hypersurface $S$ and $\eta^{\epsilon \alpha \beta \nu}$ the spacetime volume 4 -form, the constraints are:

$$
\begin{aligned}
n^{\alpha}\left(\nabla^{\beta} K_{\alpha \beta \lambda \mu}-J_{\alpha \lambda \mu}\right) & =0, \\
n_{\nu} \eta^{\epsilon \alpha \beta \nu} \nabla_{\epsilon} K_{\alpha \beta \lambda \mu} & =0,
\end{aligned}
$$

where all the derivatives appearing in these expressions are tangent to $S$. This set of constraints is complete (there are 12 equations) and also integrable provided that $\nabla^{\alpha} J_{\alpha \lambda \mu}=0$, which holds because of the Einstein field equations.

Taking into account (7), equation (6) immediately implies

$$
t^{[\delta} \nabla_{\nu} K^{\gamma] \nu \alpha \beta}-\frac{3}{2} t_{\epsilon} \nabla^{[\epsilon} K^{\gamma \delta] \alpha \beta}-t^{[\delta} J^{\gamma] \alpha \beta}=0 .
$$

If we multiply the previous expression by $t_{\delta}$, we obtain $P_{\alpha}^{\sigma}\left(\nabla^{\beta} K_{\sigma \beta \lambda \mu}-J_{\sigma \lambda \mu}\right)=0$, where $P_{\alpha}^{\sigma}$ is the orthogonal projector to $t_{\alpha}$. Taking into account (8) and the fact that $t_{\alpha} n^{\alpha} \neq 0$, we obtain equation (3). Equation (四) is achieved in a similar way: we first multiply (10) by $\eta_{\gamma \delta \sigma \rho} t^{\rho}$ obtaining $P_{\nu}^{\sigma} \eta^{\epsilon \alpha \beta \nu} \nabla_{\epsilon} K_{\alpha \beta \lambda \mu}=0$, and then we follow the same reasoning as before, but now making use of constraint (9)). This shows that the set of equations (6,8,9) is equivalent to (3,4).

\section{Riemann symmetries are conserved}

Until now, we have only dealt with double 2-forms, but, actually, we are interested in the Riemann tensor. Our purpose now is to show that Bianchi equations preserve the full Riemann symmetries. The complete set of Riemann symmetries is $K_{\alpha \beta \lambda \mu}=$ 
$K_{[\alpha \beta][\lambda \mu]}$ and $K_{[\alpha \beta \lambda] \mu}=0$. In order to construct an appropriate system of differential equations, let us set the following definitions:

$$
\Xi_{\alpha \beta \lambda \mu} \equiv K_{[\alpha \beta \lambda] \mu}, \quad \Sigma_{\alpha \beta} \equiv K_{\alpha \beta}-\left(T_{\alpha \beta}-\frac{1}{2} T g_{\alpha \beta}\right)
$$

where $K_{\alpha \beta} \equiv K_{\alpha \sigma \beta}^{\sigma}$. Then we extract, from equations (3, (14) and the energy-momentum conservation equation $\nabla^{\beta} T_{\alpha \beta}=0$, the following system for the previous defined variables $\Xi_{\alpha \beta \lambda \mu}$ and $\Sigma_{\alpha \beta}$ :

$$
\begin{aligned}
\nabla^{\alpha} \Xi_{\alpha \beta \lambda \mu}+\frac{2}{3} \nabla_{[\beta} \Sigma_{\lambda] \mu} & =0 \\
\nabla^{\alpha} \Sigma_{\alpha \beta} & =0 \\
\nabla^{\beta} \Sigma_{\alpha \beta}-\frac{1}{2} \nabla_{\alpha} \Sigma & =0 .
\end{aligned}
$$

These are 32 equations for 32 unknowns forming a linear first-order homogeneous system whose only characteristic is the null cone. Now, we suppose that initially the double 2-form $K_{\alpha \beta \lambda \mu}$ satisfies the full Riemann symmetries $\left(\Xi_{\alpha \beta \lambda \mu}=0\right)$ and that, in addition, $K_{\alpha \beta}$ satisfies Einstein's equations in the sense $\Sigma_{\alpha \beta}=0$. These initial conditions have no other solution but $\Xi_{\alpha \beta \lambda \mu}=\Sigma_{\alpha \beta}=0$ (see [4, for instance) and, therefore, the Riemann symmetries, likewise the Einstein field equations, are preserved by the Bianchi equations.

\section{Acknowledgments}

We wish to thank Carlos F. Sopuerta and Sergi R. Hildebrandt for a careful reading of this manuscript. We also acknowledge the Comissionat per a Universitats $i$ Recerca de la Generalitat de Catalunya for financial support.

\section{A Positivity property of Bel tensor}

To prove that $n_{\epsilon} \mathbf{Q}^{\epsilon}$ is a positive-definite quadratic form we will make use of the standard spinor calculus [8]. First, taking into account the symmetries of $K_{\alpha \beta \lambda \mu}$, we decompose it as follows:

$$
\begin{array}{r}
K_{\nu \mu \sigma \rho}=\chi_{N M S R} \epsilon_{N^{\prime} M^{\prime}} \epsilon_{S^{\prime} R^{\prime}}+\Psi_{N M S^{\prime} R^{\prime}} \epsilon_{N^{\prime} M^{\prime}} \epsilon_{S R} \\
+\bar{\chi}_{N^{\prime} M^{\prime} S^{\prime} R^{\prime}} \epsilon_{N M} \epsilon_{S R}+\bar{\Psi}_{N^{\prime} M^{\prime} S R} \epsilon_{N M} \epsilon_{S^{\prime} R^{\prime}}
\end{array}
$$

where

$$
\chi_{N M S R}=\chi_{(N M)(S R)}, \Psi_{N M S^{\prime} R^{\prime}}=\Psi_{(N M)\left(S^{\prime} R^{\prime}\right)} .
$$


Now we proceed to calculate

$$
\begin{aligned}
& n_{\epsilon} Q^{\epsilon\left\{\gamma^{\prime} \delta^{\prime} \sigma^{\prime} \rho^{\prime}\right\}\{\gamma \delta \sigma \rho\}} K_{\gamma \delta \sigma \rho} K_{\gamma^{\prime} \delta^{\prime} \sigma^{\prime} \rho^{\prime}}=\left(K_{\nu}^{\alpha \beta}{ }_{\sigma} K_{\alpha \mu \beta \rho}+K_{\nu}^{\alpha \beta}{ }_{\rho} K_{\alpha \mu \beta \sigma}-\right. \\
& \left.-\frac{1}{2} g_{\nu \mu} K_{\sigma}^{\alpha \beta \gamma} K_{\alpha \beta \gamma \rho}-\frac{1}{2} g_{\sigma \rho} K_{\nu}^{\alpha \beta \gamma} K_{\mu \alpha \beta \gamma}+\frac{1}{8} g_{\nu \mu} g_{\sigma \rho} K_{\alpha \beta \gamma \delta} K^{\alpha \beta \gamma \delta}\right) n^{\nu} t^{\mu} u^{\sigma} v^{\rho} \text {, }
\end{aligned}
$$

which is the Bel tensor [9] for the double 2-form $K_{\alpha \beta \gamma \delta}$ contracted with four timelike future-directed vectors, $\vec{n}, \vec{t}, \vec{u}$ and $\vec{v}$. In fact, this is a little more general since we have not required symmetry between indices $\alpha \beta$ and $\lambda \mu$. In order to express (12) in spinor form it is more convenient to rewrite it in the following form:

$$
\begin{aligned}
& \frac{1}{2}\left(K_{\nu{ }_{\sigma}}^{\alpha \beta} K_{\alpha \mu \beta \rho}+K *_{\nu{ }_{\sigma}}^{\alpha \beta} K *_{\alpha \mu \beta \rho}+\right. \\
& \left.+* K_{\nu{ }^{\alpha}}^{\alpha \beta} * K_{\alpha \mu \beta \rho}+* K *_{\nu}^{\alpha \beta} * K *_{\alpha \mu \beta \rho}\right) n^{\nu} t^{\mu} u^{\sigma} v^{\rho} \text {, }
\end{aligned}
$$

and now, the dual terms appearing in this expression have simple spinor expressions (see [8]). Performing the calculations we finally obtain

$$
\begin{array}{r}
n_{\epsilon} Q^{\epsilon\{\gamma \delta \sigma \rho\}\left\{\gamma^{\prime} \delta^{\prime} \sigma^{\prime} \rho^{\prime}\right\}} K_{\gamma \delta \sigma \rho} K_{\gamma^{\prime} \delta^{\prime} \sigma^{\prime} \rho^{\prime}}= \\
=4\left(\chi_{N M S R} \bar{\chi}_{N^{\prime} M^{\prime} S^{\prime} R^{\prime}}+\Psi_{N M S^{\prime} R^{\prime}} \bar{\Psi}_{N^{\prime} M^{\prime} S R}\right) n^{\nu} t^{\mu} u^{\sigma} v^{\rho},
\end{array}
$$

which is always positive, and zero if and only if $\chi_{N M S R}=\Psi_{N M S^{\prime} R^{\prime}}=0$. This is proven by using usual methods (see, for instance, [8, 10]): let us consider a spinor basis $\left(\iota_{A}, o_{A}\right)$ and its two associated null future-directed vectors, $l_{\alpha}=\iota_{A} \bar{\iota}_{A^{\prime}}$ and $k_{\alpha}=o_{A} \bar{o}_{A^{\prime}}$. Given a timelike future-pointing vector $\vec{z}$ and a future-directed null vector $\vec{x}$, there exists another future-directed null vector $\vec{y}$ such that:

$$
\vec{z}=a \vec{x}+\vec{y}=a x_{A} \bar{x}_{A^{\prime}}+y_{A} \bar{y}_{A^{\prime}}, a>0 .
$$

Applying this decomposition to $\vec{n}, \vec{t}, \vec{u}$ and $\vec{v}$, (13) becomes a sum of positive terms, so the positivity is shown. Suppose now that the expression (13) is zero. We can decompose the four previous vectors in many differents ways by choosing their corresponding

vector $\vec{x}$ to be $\vec{l}$ or $\vec{k}$. Then, for every choice we do, among the positive terms of the sum we will get a component of $\chi_{N M S R}$ and $\Psi_{N M S^{\prime} R^{\prime}}$ in the spinor basis mentioned above. Therefore, making all the possible choices, we will obtain all the components zero, which means that $K_{\alpha \beta \lambda \mu}=0$.

\section{References}

[1] Bona C, Masso J, Seidel E and Stela J 1995 A new formalism for numerical relativity Phys. Rev. Lett. 75 600-3

[2] Geroch R 1995 Partial Differential Equations of Physics, in General Relativity ed G S Hall and J R Pulham (SUSSP Publications and IoP) Preprint gr-qc/9602055 
[3] Friedrichs K O 1954 Symmetric hyperbolic linear differential equations Commun. Pure Appl. Math. 7 345-92

[4] John F 1982 Partial Differential Equations (New York: Springer)

[5] Friedrich H 1996 Hyperbolic reductions for Einstein's equations Class. Quantum Grav. 13 1451-69

[6] Anderson A, Choquet-Bruhat Y and York J W Jr 1997 Einstein-Bianchi Hyperbolic System for General Relativity (to appear in Topological Methods in Nonlinear Analysis) Preprint gr-qc/9710041

[7] Lichnerowicz A 1960 Ondes et radiations électromagnétiques et gravitationelles en relativité générale Annali di matematica pura ed applicata 50 1-96

[8] Penrose R and Rindler W 1986 Spinors and Space-Time (Cambridge: Cambridge University Press)

[9] Bel L 1959 Introduction d'un tenseur du quatrième ordre C.R. Acad. Sci. 248 $1297-1300$

[10] Bergqvist G 1998 Positivity properties of the Bel-Robinson tensor J. Math. Phys. $392141-7$ 